\title{
Sensor-Assisted Cooperative Localization and Communication in Multi-agent Networks
}

\author{
Mattia Brambilla
}

\begin{abstract}
This brief highlights research advances on cooperative techniques for localization and communication. These two macro trends are investigated in the general context of mobile multi-agent networks for situational awareness applications, where time-varying agents of unknown locations are asked to fulfill positioning and information sharing tasks. Cooperative localization is conceived for both active and passive agents, i.e., targets to be detected and localized, and it is analyzed in vehicular and maritime environments. Communication is investigated for vehicular scenarios, where vehicles are requested to share massive data in the perspective development of connected and automated mobility systems. Both research areas rely on the integration of heterogeneous sensors and communication. Specifically, it is studied how to improve localization by exploring communication techniques as well as how to enhance communication performances by extracting information from perception sensors. The dynamic environment of multi-agent systems calls for robust, flexible and adaptive techniques, capable of profitably fuse different types of information, and the outcomes of these researches show how a statistical approach based on cooperation guarantees higher resilience, reliability and confidence.
\end{abstract}

\section{Introduction}

This chapter summarizes the main research works characterizing my Ph.D. studies at Politecnico di Milano from Nov. 2017 until Oct. 2020, under the supervision of Professor Monica Nicoli, which lead to the doctoral dissertation in [1] and the scientific publications in [2-12].

In the context of multi-agent networks, this chapter discusses research advances on cooperative localization and communication among time-varying agents of unknown positions. These two tasks (localization and communication) may sound as independent and disjoint, however the digital evolution of communication technologies and

\footnotetext{
M. Brambilla $(\bowtie)$

Dipartimento di Elettronica, Informazione e Bioingegneria, Politecnico di Milano, Via Ponzio 34/5, 20133 Milano, Italy

e-mail: mattia.brambilla@polimi.it

(C) The Author(s) 2022

L. Piroddi (ed.), Special Topics in Information Technology,

PoliMI SpringerBriefs, https://doi.org/10.1007/978-3-030-85918-3_11
} 
hardware equipment enables their fruitful integration, leading them to mutually assist each other.

The intuition that communication and localization are going to converge can be found in the activities of standardization groups for 5G, with the introduction of specific positioning-related signals in the $3 \mathrm{GPP}^{1}$ Release 16 . The trend gets higher attention for next releases of the communication standard (i.e., 6G), where a shift towards high-frequency communications will allow to extract accurate information from the environment and provide precise situational awareness for the user. The use of signals at millimeter-wave $(30-100 \mathrm{GHz})$, sub-terahertz $(100-300 \mathrm{GHz})$, terahertz $(300 \mathrm{GHz}-10 \mathrm{THz})$ and optical $(>10 \mathrm{THz})$ frequencies defines new frontiers for short-range wireless communications, opening poorly-explored areas over large scale and introducing new challenges for hardware, algorithms and protocol design.

Integration goes together with cooperation, as multiple connected devices (such as vehicles) engage into an evolving network characterized by intermittent links due to mobility. This scenario paves the way for cooperative processing algorithms where multiple types of information/data/measurements need to be constructively handled for a coherent analysis.

In the following, cooperative localization is addressed in Sect. 2, where a general formulation of the whole problem is given by specifically defining the modeling assumptions (Sect. 2.1) and the types of measurements (Sect. 2.2), followed by the conceptualization of the methodology (Sect. 2.3) and the definition of analyzed scenarios (Sect. 2.4). The topic of communication, instead, is discussed for the vehicular environment in Sect. 3, where beam-based communications are investigated. A same structure is used, starting from the main assumptions (Sect. 3.1), passing through the specification of vehicle measurements (Sect. 3.2), until the discussion on the methodology (Sect. 3.3) and simulation scenarios (Sect. 3.4). Extra research topics of my $\mathrm{Ph} . \mathrm{D}$. are referenced in Sect. 4. Concluding remarks are drawn in Sect. 5.

\section{Cooperative Localization}

The first major research topic of my Ph.D. studies covers the area of cooperative localization, and most of the contents have been published in [2-4]. A network of multiple agents has the advantage of overcoming the ego-agent capability by enlarging the set of available information. The performance of an individual localization system is limited both in terms of field of view and accuracy, as it can only rely on the information coming from a single device. On the other hand, connected agents can acquire more information from different perspectives, thus being able to provide a more accurate positioning. Localizing agents in a network refers to the capability of estimating their positions (and possibly other kinematics or non-kinematics parameters). This task involves both cooperative (which intentionally share information) and non-cooperative agents, the latter are called targets. The time-variant network

\footnotetext{
${ }^{1}$ Third Generation Partnership Project.
} 


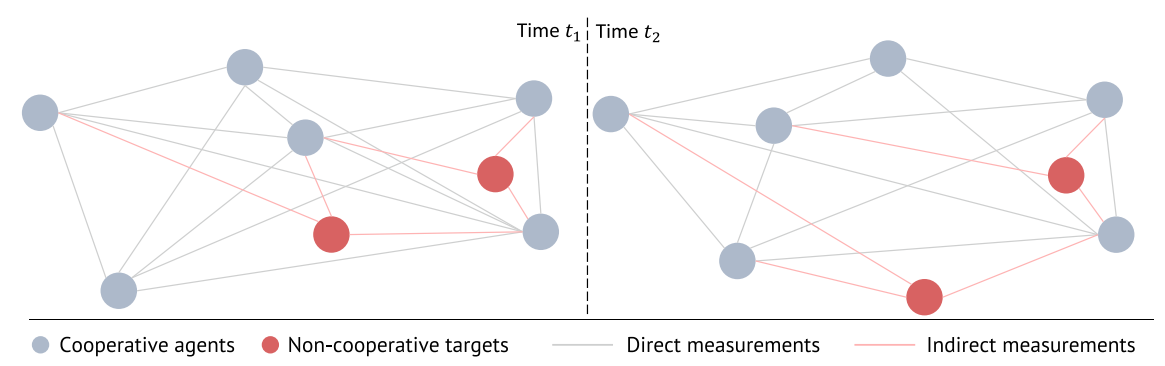

Fig. 1 Illustrative example of a time-varying topology of a generic multi-agent network of cooperative agents and non-cooperative targets. From time step $t_{1}$ to $t_{2}$, the mobility of agents and targets, as well as the availability of connections/measurements define two different network topologies

topology is created according to the agent/target dynamics (i.e., if they are static or moving) together with the availability/non-availability of connections among them. These concepts are graphically illustrated in Fig. 1, where an example of network topology variation between two time steps is provided.

\subsection{Cooperative Localization: Assumptions}

The research on cooperative localization is carried out under the following main conceptual assumptions (related to the system model). Agents and targets are assumed to be point objects in a 2D space, i.e., neglecting physical dimension and occupancy. It follows that the state of an agent comprises the position (and velocity) of the corresponding point. Practically, this choice can be referred to the use of the barycenter of the object itself. Ignoring the vertical dimension, instead, reduces the scene to be depicted over a plane, meaning that all agents and targets have a same height. All measurements are thus computed in a $2 \mathrm{D}$ domain with respect to the point representations, and they are assumed to be unique, i.e., it is not possible to have more than one data per each time step measuring a same quantity. It follows that an agent can have up to one measurement of each type (see next section) for itself and each nearby agent/target at each specific time step. As far as the algorithmic part is concerned, many other specific assumptions are considered but, to avoid to go into technicalities, they are omitted here. For details, please refer to [1, Ch. 3-4].

\subsection{Cooperative Localization: Measurements}

Cooperative localization of agents and targets is enabled by the use of measurements. In a general problem formulation, it is possible to classify measurements into three categories as follows: 
- Ego measurement: it refers to a measurement of an agent made by the agent itself. The measurement can be related to the full agent state or part of it. As an example, a GPS ${ }^{2}$ measurement estimates the position of the agent. If the agent state comprises only the position (e.g., latitude/longitude) the GPS information is truly defining the full agent state, if the agent state includes additional parameters (e.g., the velocity), the GPS data is just a partial observation of the agent state.

- Direct measurement: it refers to a relative measurement of an agent with respect to another one. Distance and angular measurements fall within this category. An agent is able to estimate the relative distance or bearing angle with respect to another one by decoding known signals. Specifically, considering a pair of agent where one plays the role of transmitter (Tx) and the other of receiver (Rx), the $\mathrm{Rx}$-agent can retrieve its distance information with respect to the $\mathrm{Tx}$ by measuring the time difference between the signal generation and reception. Similarly, by estimating the direction of arrival of the received signal, the Rx-agent can determine the relative bearing with respect to the Tx one.

- Indirect measurement: it refers to a measurement of a target made by an agent. This type of measurements is used to localize non-cooperative targets that do not deliberately transmit a signal. Despite being similar to a direct measurement in the sense of letting an Rx-agent extracting range and bearing from a known signal, they significantly differ from the former as the measured quantities refer to an hypothesized target. This means that the Rx-agent has to decode a known signal sent from a Tx-agent, and discriminate between the direct path (i.e., the direct measurement) and the reflections. Identifying the source of reflections points let the Rx-agent determine the presence of unknown entities (i.e., the targets) in an indirect way. In this process, only first order reflections of the signal are used.

Note that a same known Tx signal can be exploited for both direct and indirect measurements, and the Rx capability of signal processing for multipath identification allows the distinction between direct and indirect measurement. Note also that $\mathrm{Tx}$ and Rx agents can coincide, meaning that the agent intentionally transmit a known signal for target detection and localization (in this specific case, a direct measurement does not exist). Technically, if Tx and Rx agents coincide, the configuration is monostatic, while if they differ it is bistatic.

\subsection{Cooperative Localization: Methodology}

The problem of cooperative localization of agents and targets by exploring the shared set of measurements described in Sect. 2.2 under the assumptions in Sect. 2.1 is addressed by developing a Bayesian filtering approach. The message passing scheme

\footnotetext{
${ }^{2}$ GPS-Global Positioning System.
} 
of the sum-product algorithm (or belief propagation [13, 14]) is the algorithmic fundamental, in which measurements are converted into messages that bounce from agent-related, target-related and measurement-related variables in a loopy way and iteratively refine the agent/target beliefs. A belief is the estimation of agent/target marginal probability density distribution (e.g., the spatial area in which it is likely to be located). Message passing algorithms make use of a graphical representation of the multi-agent network to solve the considered inference problem, incorporating multiple variables into one unique framework. Graph-based models are suitable for capturing the intrinsic network-like structure of the multi-agent network, where agents and targets play the roles of nodes, while the connections among them indicate the availability of communication links and measurements. Such formulation has the advantage of fitting both centralized and distributed architectures. In the latter case, it allows for parallel information exchange (messages), speeding up the flooding of information (at each node) to rapidly reach all nodes in few multi-hop exchanges.

The proposed solution considers the localization of cooperative agents and the multi-target tracking as two integrated tasks. Compared to state-of-the-art solutions [15-19], the developed methodology integrates the uncertainties of agents (coming from the need of localizing them) with the tracking of unknown and arbitrary number of targets, and fruitfully exploits target localization as a mean to improve agent self-localization. The idea can be conceptualized as a dual layer mutually-connected graph (see Fig.2), where the two tasks are indicated over two distinct planes but the upside-down connections among agents and targets denote a mutual exchange of information. Ego and direct measurements are used for the top layer (cooperative agent localization) only, while indirect measurements define the inter-layer connections and enable the multi-target tracking.

The proposed general formulation manages the agent/target uncertainties as well as communication failures (i.e., intermittent measurement availability) and typical multitarget tracking challenges like the target birth and death, the presence of clut-

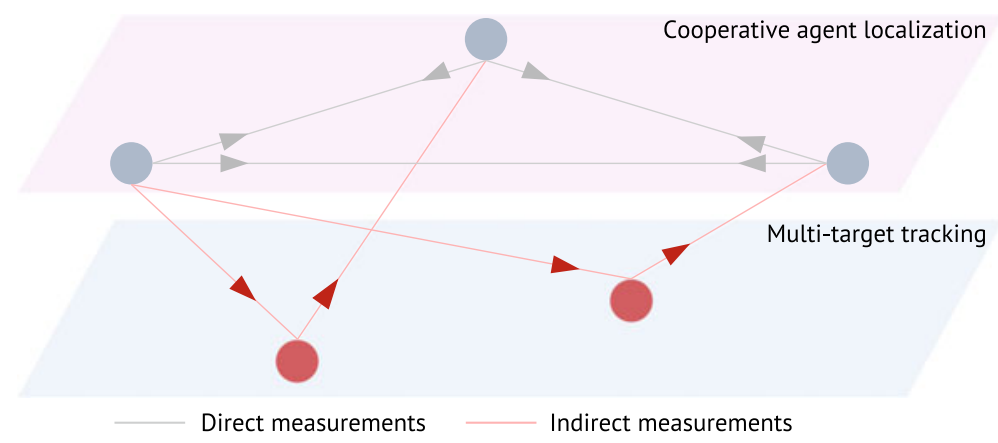

Fig. 2 Conceptualization of the integrated tasks of cooperative agent localization and multi-target tracking, which are unified in a single graph where messages (indicated as directed arrows) are flooded among agents and targets. The directions of the arrows from targets to agents indicate that target-related information is extracted and used to refine the beliefs of agents 
ter measurements (i.e., false alarms), missed detections, and measurement origin uncertainty (i.e., the problem of unknown association between targets and indirect measurements). All details concerning the general stochastic modeling and algorithmic properties are detailed in the doctoral dissertation [1, Ch. 3].

\subsection{Cooperative Localization: Application Scenarios}

The developed solution for cooperative agent localization and multitarget tracking is general enough to accommodate a full multistatic network configuration, with multiple transmitters and receivers, thus guaranteeing a wide range of applications in safety-related and situational awareness contexts.

In my Ph.D. studies, researches have been focused on maritime and vehicular environments. In the former case (see [1, Ch. 3]), agents can be identified as autonomous underwater vehicles, ships/vessels, sea sensors, buoys or wave gliders, while targets can be intruding/illegal ships, military vessels or other types of potential threats. In the latter case (see [1, Ch. 4]), agents can be land vehicles, while targets can refer to vulnerable road users or other non-connected vehicles. Each environment has its own peculiarities, especially related to the agent/target mobility, technology, measurement availability and accuracy. Nevertheless, the proposed solution is flexible enough to accommodate for specific needs of each scenario, and it guarantees a high degree of adaptability.

The vehicular environment has been considered for the first analyses, and the achieved results considered a simplified algorithm for multitarget tracking without clutter and missed detections, with a known set of possible targets to be localized, and without direct measurements among vehicles. On the other hand, both centralized and distributed implementations have been tested, showing that a fully distributed vehicular network with direct communication links among vehicles can achieve a same performance as the case of a centralized architecture. In this scenario ego measurements are available through GPS, while target localization can be performed by processing on-board perception sensors (e.g., radar, lidar, camera), and the communications can exploit wireless links (e.g., cellular technology). The assessments through simulations consider both simplified scenes and realistic urban traffic flow conditions over a real road network, where two distinct mobility scenarios (a traffic light regulated case and a fully autonomous one) are taken into account. Performance results highlight the potential impact of the proposed technique for next generation mobility systems, showing enhancements with respect to GPS-based positioning.

The maritime environment has been studied as a second research area, where the generalized version of the proposed methodology has been truly applied for a centralized network architecture. This research comes from a collaboration with the NATO STO CMRE (North Atlantic Treaty Organization-Science and Technology Organisation-Centre for Maritime Research and Experimentation). In this scenario, ego measurements can be available through GPS in case of surface agents, while inertial information should be used in case of underwater agents. Due to water 
medium attenuation, in fact, it is only possible to use acoustic communication links to exchange information in the underwater domain. The same acoustic signal is used for direct and indirect measurements. The achieved results highlight the algorithm resilience to handle a time-varying number of mobile targets and extract from them a valuable information to improve the localization of agents, despite the challenging bistatic settings. It has been proven through simulations how the target implicit information is of utmost importance in case of agent outage conditions as it still allows to satisfactory localize lost agents. In the Ph.D. thesis, a simulated maritime surveillance use case is analyzed (see [1, Ch. 3]), but an assessment over real underwater data is going to be released too.

\section{Vehicular Communication}

The second major research topic of my Ph.D. studies covers the area of communication in next-generation vehicular networks, and most of the contents have been published in [5-10]. The need of letting vehicle share massive amount of data comes from the evolution towards connected and automated mobility systems, where a set of services are going to be available for users, improving road safety and transportation efficiency [20, 21]. Vehicle-to-Everything (V2X) communications require technologies, standards and protocols for the development of a connected mobility ecosystem. Vehicle-to-Vehicle (V2V) and Vehicle-to-Infrastructure (V2I) fall within the V2X umbrella. As the definitions may suggest, these specific types of wireless communications allow a vehicle to connect with nearby road players such as other vehicles $(\mathrm{V} 2 \mathrm{~V})$, road infrastructure (V2I) or other types of actors like pedestrians (technically a device handled by a pedestrian, e.g., a smartphone). The research on vehicular communication is centered on beam-based communications, where vehicles use directive narrow beams to confine the signal with the assistance of on-board sensors (see Fig. 3). Two communication technologies are analyzed and compared, namely millimeter Wave (mmWave) and Free-Space Optics (FSO).

\subsection{Vehicular Communication: Assumptions}

This research addresses the currently emerging area of V2X communication, which is a rapidly-evolving field where brand new ideas are being proposed, also confirmed by an increasing interest from both research and industry perspectives, due to the needs of developing ad-hoc solutions to meet a constantly growing market demand. The addressed V2X-related research started from simplistic models of vehicles and trajectories, until reaching nearly realistic simulation settings. As a matter of facts, first analyses dealt with a $2 \mathrm{D}$ vehicle model $[5,6]$, as they were focused on preliminary assessment and validation of the impact of vertical vehicle vibrations (due to driving style and road pavement). Motivated by promising results and feedbacks, the 

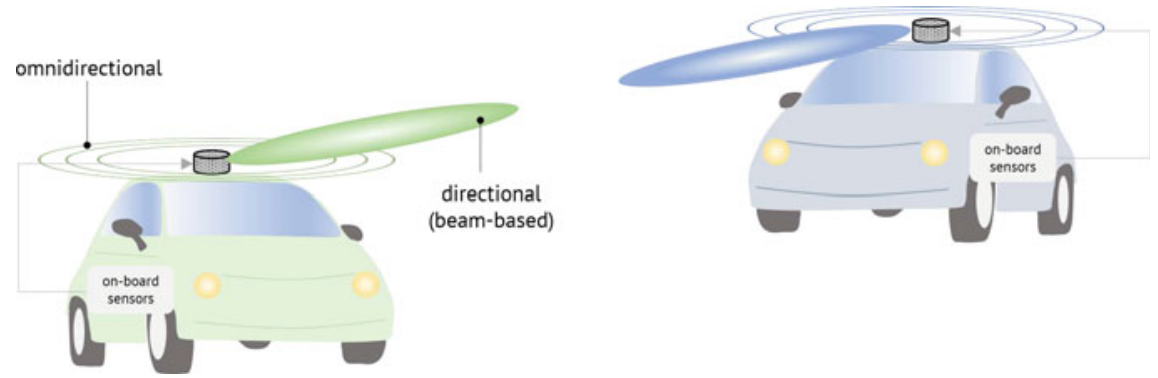

Fig. 3 Illustration of vehicular omnidirectional and beam-based communications. A connection with telecommunication apparatus and on-board sensors is also shown to highlight the concept of the proposed solution

complexity of system model has been increased by introducing a full 3D description of vehicle position and orientation [7, 8]. This generalization allowed more in-dept analyses on the degradation effects due to beam mis-alignment as a consequence of the combined effects of position and orientation errors, vehicle motion trajectory and road pavement imperfections. From a technological perspective, mmWave and FSO communication technology are simulated by assuming steerable devices able to irradiate in any 3D direction, and the $\mathrm{V} 2 \mathrm{~V}$ channels are always assumed as ideal (i.e., without obstructions, shadowing, fading, reflections, etc.). Indeed, the research focuses on the analysis of the degrading impact of misalignment on beam-based communications, thus separating the effects of signal transmission with the ones merely due to incorrect beam pointing.

\subsection{Vehicular Communication: Measurements}

The proposed sensor-assisted methodology for vehicular communications relies on the ego-vehicle knowledge of its position and orientation, which are used to control the beam. A vehicle uses on-board GPS and Inertial Measurements Unit (IMU) to retrieve its own position and orientation, to be possibly integrated in a navigation filter for a more robust estimation. The processed state information is locally used to adjust the beam pointing direction, i.e., by keeping the desired pointing regardless of the vehicle motion. Vehicle position information is also shared (over a reliable communication link) to enable cooperation with connected vehicles (which perform dual operations). Information sharing is thus the enabler of the cooperative sensorassisted beam alignment scheme which is discussed in my doctoral dissertation [1, Ch. 5]. Therein, a system level architectural vision is also highlighted, offering a more general framing of the proposed solution in a realistic V2X connected mobility ecosystem. In particular, it is mentioned how it could be possible to integrate vehicle perception sensors (e.g., radar, lidar, camera) in an extension of the proposed solution. 


\subsection{Vehicular Communication: Methodology}

Communicating at narrow beams is currently deemed as the most promising technological solution able to meet high-data rate requirements for advanced autonomous automotive systems [22-25]. This research focuses on the use of on-board sensors (that precisely estimate the vehicle position and orientation) to assist the pointing of the beam. As communication involve two entities (a transmitter and a receiver), it is proposed to cooperatively share the vehicle state for a seamless and dynamic beam pointing, able to dynamically compensate for the reciprocal motion of vehicles. Being able to proactively known the position of the communication counterpart allows a vehicle to determine the candidate pointing direction without iteratively searching for the best candidate beam, thus saving time and improving the communication efficiency. Moreover, if position information comes with the associated accuracy, a vehicle can also implement a beamwidth and power adaptation algorithm, where not only the pointing direction is dynamically computed, but also the size of the beam and the required transmission power are optimally adjusted [10]. The exchange of vehicle navigation information is used as enabler to fasten the alignment of narrow beams such that the higher data rates of high frequency (mmWave and FSO) links are fully exploited.

Most of the research work is focused on the V2V link degradation due to beam misalignment as a consequence of vehicle mobility [5-8, 10], but a study on V2I channel modeling has also been carried out to evaluated potential benefits of exploiting mmWave sparsity to design a robust beam alignment [9].

\subsection{Vehicular Communication: Application Scenarios}

To evaluate the performance of the proposed sensor-assisted solution for beam-based vehicular communications, different simulation scenarios have been considered. As a starting point, a straight road use case has been the first considered environment for $2 \mathrm{D}$ evaluation $[5,6]$. It has then been extended to account for full 3D mobility over a winding trajectory [7,8]. Analyses not included in the thesis but carried out in the last period of my Ph.D., instead, considered a set of collected mobility data [10]. In all cases, despite considering different environments, simulation conditions, technologies and assumptions, it has been shown that the proposed sensorassisted beam-based (mmWave or FSO) solution for V2V communications brings remarkable improvements in terms of higher throughput, less outage and reduced signaling, providing an alternative to the onerous exhaustive beam search which does not use any information from sensors [1, Ch. 5]. 


\section{Further Research Topics}

During my Ph.D., I have also worked on extra topics that are not included in the doctoral dissertation. The interested reader in localization and tracking experimentation for industry application based on ultra wide band technology can find details in [11, 12].

\section{Concluding Remarks}

In this chapter, I summarized the major contents and outcomes of my doctoral research on sensor-assisted techniques for cooperative localization and communication. Multi-agent networks are the targeted use cases of this research, with main (but not exclusive) applications to maritime and vehicular contexts.

In the field of cooperative localization, graph theory has been used to describe the relations among agents, where the existence/absence of links allows/precludes the exchange of information, enabling a robust/weak data fusion for localization. Both centralized and distributed implementations have been considered, where the distinction reflects the specific targeted applications. Fully distributed solutions better suit vehicular networks, which are characterized by fast (order of seconds or less) variation in network topology, and where latency constraints call for decentralized processing at vehicles. On the other hand, in maritime surveillance, a different time scale (order of minutes) facilitates a centralized solution, where data from multiple agents/sensors are collected in a single fusion center. Moreover, processing offload is preferred due to intrinsic autonomy limitation of battery-powered vehicles, which are preferable to be long-lasting sensors rather than fast-processing units.

In the field of vehicular communication, it has been proposed to integrate on-board (already available) sensors to assist and enhance the communication performance. Guaranteeing a stable and reliable V2X link allows vehicles to implement advanced driving functionalities towards fully connected and automated mobility. It is stressed how the integration of two engineering domains (automotive and telecommunication) is highly recommended for the evolution of connected mobility services.

Recalling the introduction of this chapter, an interesting, pivotal and of utmost interest research direction would be the integration of the two fields in one joint localization and communication solution in the perspective of $6 \mathrm{G}$ wireless systems for integrated mobility over multiple domains, suitable for both centralized and distributed implementations. 6G wireless systems aim at the convergence of communication, sensing and localization in one integrated platform connecting multiple agents of diverse types, hardware and requirements, and the research topics in my Ph.D. thesis might inspire ideas for the standardization of new protocols and algorithms. 


\section{References}

1. M. Brambilla, Sensor-assisted cooperative localization and communication in multi-agent networks. PhD dissertation, Politecnico di Milano (2021) doi: hdl.handle.net/10589/170700

2. M. Brambilla, G.M. Soatti, M. Nicoli, Precise vehicle positioning by cooperative feature association and tracking in vehicular networks, in IEEE Statistical Signal Processing Workshop (SSP) (2018), pp. 648-652. https://doi.org/10.1109/SSP.2018.8450794

3. M. Brambilla, M. Nicoli, G. Soatti, F. Deflorio, Augmenting vehicle localization by cooperative sensing of the driving environment: insight on data association in urban traffic scenarios. IEEE Trans. Intelligent Transp. Syst. 21(4), 1646-1663 (2020). https://doi.org/10.1109/TITS.2019. 2941435

4. R. Mendrzik, M. Brambilla, C. Allmann, M. Nicoli, W. Koch, G. Bauch, K. LePage, P. Braca, Joint multitarget tracking and dynamic network localization in the underwater domain, in IEEE International Conference on Acoustics, Speech and Signal Processing (ICASSP) (2020), pp. 4890-4894. https://doi.org/10.1109/ICASSP40776.2020.9054047

5. M. Brambilla, M. Nicoli, S. Savaresi, U. Spagnolini, Inertial sensor aided mmWave beam tracking to support cooperative autonomous driving, in IEEE International Conference on Communications Workshops (ICC Workshops) (2019), pp. 1-6. https://doi.org/10.1109/ICCW. 2019.8756931

6. M. Brambilla, A. Matera, D. Tagliaferri, M. Nicoli, U. Spagnolini, RF-assisted free-space optics for $5 \mathrm{G}$ vehicle-to-vehicle communications, in IEEE International Conference on Communications Workshops (ICC Workshops) (2019), pp. 1-6. https://doi.org/10.1109/ICCW. 2019.8757059

7. M. Brambilla, D. Tagliaferri, M. Nicoli, U. Spagnolini, Sensor and map-aided cooperative beam tracking for optical V2V communications, in IEEE 91st Vehicular Technology Conference (VTC2020-Spring) (2020), pp. 1-7. https://doi.org/10.1109/VTC2020-Spring48590. 2020.9129590

8. M. Brambilla, L. Combi, A. Matera, D. Tagliaferri, M. Nicoli, U. Spagnolini, Sensor-aided V2X beam tracking for connected automated driving: distributed architecture and processing algorithms. Sensors 20(12) (2020). https://doi.org/10.3390/s20123573

9. M. Brambilla, D. Pardo, M. Nicoli, Location-assisted subspace-based beam alignment in LOS/NLOS mm-wave V2X communications, in IEEE International Conference on Communications (ICC) (2020), pp. 1-6. https://doi.org/10.1109/ICC40277.2020.9148587

10. D. Tagliaferri, M. Brambilla, M. Nicoli, U. Spagnolini, Sensor-aided beamwidth and power control for next generation vehicular communications. IEEE Access 9, 56301-56317 (2021). https://doi.org/10.1109/ACCESS.2021.3071726

11. L. Barbieri, M. Brambilla, R. Pitic, A. Trabattoni, S. Mervic, M. Nicoli, UWB real-time location systems for smart factory: augmentation methods and experiments, in IEEE 31st Annual International Symposium on Personal, Indoor and Mobile Radio Communications (2020), pp. 1-7. https://doi.org/10.1109/PIMRC48278.2020.9217307

12. L. Barbieri, R. Brambilla, A. Trabattoni, S. Mervic, M. Nicoli, UWB localization in a smart factory: augmentation methods and experimental assessment. IEEE Trans. Instrumentation Measurement (70) (2021) https://doi.org/10.1109/TIM.2021.3074403

13. F.R. Kschischang, B.J. Frey, H.A. Loeliger, Approximate evaluation of marginal association probabilities with belief propagation. IEEE Trans. Information Theor. 47(2), 498-519 (2001). https://doi.org/10.1109/18.910572

14. J. Williams, R. Lau, Approximate evaluation of marginal association probabilities with belief propagation. IEEE Trans. Aerosp. Electronic Syst. 50(4), 2942-2959 (2014). https://doi.org/ 10.1109/TAES.2014.120568

15. V. Savic, Hlawatsch, E.G. Larsson, Target tracking in confined environments with uncertain sensor positions. IEEE Trans. Vehicular Technol. 65(2), 870-882 (2015). https://doi.org/10. 1109/TVT.2015.2404132 
16. F. Meyer, P. Braca, P. Willett, F. Hlawatsch, A scalable algorithm for tracking an unknown number of targets using multiple sensors. IEEE Trans. Signal Process. 65(13), 3478-3493 (2017). https://doi.org/10.1109/TSP.2017.2688966

17. F. Meyer, T. Kropfreiter, J. Williams, R. Lau, F. Hlawatsch, P. Braca, M.Z. Win, Message passing algorithms for scalable multitarget tracking. Proc. IEEE 106(2), 221-259 (2018). https://doi.org/10.1109/JPROC.2018.2789427

18. G. Soldi, F. Meyer, P. Braca, F. Hlawatsch, Self-tuning algorithms for multisensor-multitarget tracking using belief propagation. IEEE Trans. Signal Process. 67(15), 3922-3937 (2019). https://doi.org/10.1109/TSP.2019.2916764

19. P. Sharma, A. Saucan, D.J. Bucci, P.K. Varshney, Decentralized Gaussian filters for cooperative self-localization and multi-target tracking. IEEE Trans. Signal Process. 67(22), 5896-5911 (2019). https://doi.org/10.1109/TSP.2019.2946017

20. ETSI TR 102638 v1.1.1: Intelligent Transport Systems (ITS); Vehicular Communications; Basic Set of Applications; Definitions (2009)

21. 3GPP TS 22.186 v16.2.0: 3rd Generation Partnership Project; technical specification group services and system aspects; study on enhancement of 3GPP support for 5G V2X services (Release 16) (2019)

22. B. Bertenyi, 5G evolution: what's next? IEEE Wireless Commun. 28(1), 4-8 (2021). https:// doi.org/10.1109/MWC.2021.9363048

23. H. Tataria, M. Shafi, A.F. Molisch, M. Dohler, H. Sjöland, F. Tufvesson, 6G wireless systems: vision, requirements, challenges, insights, and opportunities. Proc. IEEE 70(7), 1166-1199 (2021). https://doi.org/10.1109/JPROC.2021.3061701

24. W. Jiang, B. Han, M.A. Habibi, H.D. Schotten, The road towards 6G: a comprehensive survey. IEEE Open J. Commun. Society 2, 334-366 (2021). https://doi.org/10.1109/OJCOMS.2021. 3057679

25. C. De Lima, D. Belot, R. Berkvens, A. Bourdoux, D. Dardari, M. Guillaud, M. Isomursu, E.S. Lohan, Y. Miao, A.N. Barreto, M.R.K. Aziz, J. Saloranta, T. Sanguanpuak, H. Sarieddeen, G. Seco-Granados, J. Suutala, T. Svensson, M. Valkama, B. Van Liempd, H. Wymeersch, Convergent communication, sensing and localization in $6 \mathrm{G}$ systems: an overview of technologies, opportunities and challenges. IEEE Access 9, 26902-26925 (2021). https://doi.org/10.1109/ ACCESS.2021.3053486

Open Access This chapter is licensed under the terms of the Creative Commons Attribution 4.0 International License (http://creativecommons.org/licenses/by/4.0/), which permits use, sharing, adaptation, distribution and reproduction in any medium or format, as long as you give appropriate credit to the original author(s) and the source, provide a link to the Creative Commons license and indicate if changes were made.

The images or other third party material in this chapter are included in the chapter's Creative Commons license, unless indicated otherwise in a credit line to the material. If material is not included in the chapter's Creative Commons license and your intended use is not permitted by statutory regulation or exceeds the permitted use, you will need to obtain permission directly from the copyright holder. 